\title{
Modelagem hidrológica e hidráulica usando o SWMM - Storm Water Management Model na bacia urbana do Riacho Fundo I - Distrito Federal
}

\author{
Hydrological and hydraulic modeling using SWMM - Storm Water Management Model in the urban \\ basin of Riacho Fundo I - Distrito Federal
}

Modelado hidrológico e hidráulico con SWMM - Storm Water Management Model en la cuenca

urbana de Riacho Fundo I - Distrito Federal

Recebido: 26/12/2020 | Revisado: 27/12/2020 | Aceito: 28/12/2020 | Publicado: 03/01/2021

\author{
Maria Elisa Leite Costa \\ ORCID: https://orcid.org/0000-0002-5477-4349 \\ Universidade de Brasília, Brasil \\ E-mail: mariaelisa@unb.br \\ Thays Mitsuko Tsuji \\ ORCID:https://orcid.org/0000-0002-6242-3557 \\ Universidade Federal de Santa Catarina, Brasil \\ thaysmitsuko@hotmail.com \\ Sérgio Koide \\ ORCID: https://orcid.org/0000-0002-0424-5748 \\ Universidade de Brasília, Brasil \\ E-mail: skoide@unb.br
}

\begin{abstract}
Resumo
Este artigo trata-se de uma modelagem hidrológica e hidráulica do sistema de drenagem urbana na Região Administrativa do Riacho Fundo I no Distrito Federal a partir dos dados de chuva e vazão monitorados em 21 eventos entre 2018 e 2019 com o modelo SWMM - Storm Water Management Model. O objetivo do trabalho foi analisar a calibração do modelo chuva-vazão para a área de estudo a fim de validar o uso da ferramenta na gestão de drenagem urbana. Foi utilizado o programa PCSWMM que dispõe do calibrador automático SRTC, que permite a variação dos parâmetros por meio da análise de incertezas. A simulação foi feita de maneira contínua, com análise de cada evento individualmente, sendo o evento do dia 12/03/19 o que apresentou as melhores funções objetivos quando calibrados (ISE= 4,64, NSE= 0,851, R²=0,865, SEE=0,466, LSE=17,4, RMSE=3,28). Com o modelo calibrado, 20 eventos foram validados e apesar de $40 \%$ deles apresentarem o NSE negativo devido a superestimação da Qpico, o modelo foi considerado satisfatório, tendo em vista que este problema pode ser atribuído a dificuldade de medição de grandes vazões. Logo, o êxito na calibração e validação do modelo hidrológicos e hidráulico proposto para a região foi atingido. Essas ferramentas são importantes para o manejo das águas pluviais, inclusive para o dimensionamento dos sistemas com medidas compensatórias.
\end{abstract}

Palavras-chave: Drenagem urbana; Calibração; Chuva; Vazão.

\begin{abstract}
This paper is about a hydrological and hydraulic modeling at the urban drainage system in the Administrative Region of Riacho Fundo I in the Federal District from the rainfall and runoff data monitored in 21 events between 2018 and 2019 with the SWMM model. The objective of the research was to analyze the calibration of the runoff model for the study area in order to validate the use of the tool in the management of urban drainage. The PCSWMM program was used, which has the SRTC automatic calibrator, which allows the variation of the parameters through the analysis of uncertainties. The simulation was carried out continuously, with the analysis of each event individually, with the event on $03 / 12 / 19$ having the best objective functions when calibrated $\left(\mathrm{ISE}=4.64, \mathrm{NSE}=0.851, \mathrm{R}^{2}=0.865, \mathrm{SEE}=0.466\right.$, LSE $=17.4$, RMSE $=3.28$ ). With the calibrated model, 20 events were validated and although $40 \%$ of them presented negative NSE due to overestimation of Qpico, the model was considered satisfactory, considering that this problem can be attributed to the difficulty of measuring large flows. Therefore, the accomplishment in the calibration and validation of the proposed hydrological and hydraulic model for the region was achieved. These tools are important for the management of rainwater, including the dimensioning of systems with compensatory measures.
\end{abstract}

Keywords: Urban drainage; Calibration; Rainfall; Flow.

\section{Resumen}

Esta investigación modeló hidrológica e hidráulicamente el sistema de drenaje urbano en la Región Administrativa de Riacho Fundo I en el Distrito Federal a partir de los datos de lluvia y caudal monitoreados en 21 eventos entre 2018 y 2019 con el modelo SWMM. El objetivo de la investigación fue analizar la calibración del modelo de escorrentía para el área de estudio con el fin de validar el uso de la herramienta en la gestión del drenaje urbano. Se utilizó el programa 
PCSWMM, que cuenta con el calibrador automático SRTC, que permite la variación de los parámetros mediante el análisis de incertidumbres. La simulación se realizó de forma continua, con el análisis de cada evento individualmente, teniendo el evento del 03/12/19 las mejores funciones objetivo al calibrarse (ISE $=4.64$, NSE $=$ 0.851, $\mathrm{R}^{2}=0.865, \mathrm{SEE}=0,466, \mathrm{LSE}=17,4, \mathrm{RMSE}=3,28$ ). Con el modelo calibrado se validaron 20 eventos y aunque el $40 \%$ de ellos presentó NSE negativo por sobreestimación de Qpico, el modelo se consideró satisfactorio, considerando que este problema se puede atribuir a la dificultad de medir grandes caudales. Por lo tanto, se logró la calibración y validación del modelo hidrológico e hidráulico propuesto para la región. Estas herramientas son importantes para la gestión del agua de lluvia, incluido el dimensionamiento de sistemas con medidas compensatorias.

Palabras clave: Drenaje urbano; Calibración; Lluvia; Caudal.

\section{Introdução}

Os sistemas de drenagem urbana convencionais impactam os corpos hídricos receptores pelo lançamento das águas pluviais, seja pelos grandes volumes ou pelas cargas de poluição difusa (Lian et al., 2019; Müller et al., 2019; Porto, 1995). As falhas causadas pela concepção higienista dessas estruturas ainda são agravadas pelo avanço da urbanização no uso e ocupação do solo (Pompêo, 1999; Silveira, 1998).

Ferramentas, como a modelagem hidrológica e hidráulica, trouxeram avanço na gestão dos sistemas de drenagem urbana. O modelo SWMM - Storm Water Management Model pode ser aplicado desde as fases de planejamento e análise dos sistemas existentes, como também no dimensionamento de novas estruturas, inclusive com a aplicação de medidas de Desenvolvimento de Baixo Impacto (Low Impact Development-LIDs) (Zhang et al., 2020; Broekhuizen et al., 2019; Xu et al., 2018; Barros et al., 2016). Além disso, o SWMM permite a simulação do fluxo nos condutos por meio da onda dinâmica, que considera os efeitos de remanso, fluxo reverso, fluxo a superfície livre e sob pressão, comum em sistemas de drenagem sobrecarregados, e gera resultados mais precisos.

O SWMM se caracteriza como um modelo dinâmico de chuva-vazão utilizado para simular o percurso dos escoamentos e cargas poluidoras gerados pelos dados de precipitações de eventos chuvosos isolados ou simulação de séries históricas. O modelo atua conforme as equações de conservação de massa e de quantidade de movimento para fluxo não permanente, que é regido pelas equações de Saint-Venant (Rossman, 2009).

Desse modo, o processo de calibração é fundamental para obtenção de um modelo com boa acurácia, a fim de que os mesmos representem os fenômenos hidráulicos e hidrológicos estudados da forma mais realística possível. Niazi et al. (2017) realizaram a calibração do SWMM manualmente, alterando um parâmetro por vez e comparando as previsões do SWMM com as observações. Este método de tentativa e erro tem sido amplamente utilizado por pesquisadores (Blumensaat et al., 2012; Chung et al., 2011; Walsh et al., 2014; Wu et al., 2013). Porém, as calibrações automáticas estão sendo utilizadas com bons resultados (Broekhuizen et al., 2019; Paule-Mercado e Lee, 2016).

O objetivo do trabalho foi analisar a calibração do modelo chuva-vazão para a área de estudo a fim de validar o uso da ferramenta na gestão de drenagem urbana. Deste modo, esta pesquisa modelou o sistema de drenagem urbana com o SWMM na Região Administrativa do Riacho Fundo I, que lança as águas pluviais no Riacho Fundo, principal afluente do Lago Paranoá, em 21 eventos que houveram monitoramento de precipitação e vazão. A bacia foi escolhida por ser uma região que possui um bom sistema de saneamento básico, inclusive sem evidências de lançamento clandestino de esgoto na rede pluvial, o que caracteriza um sistema de esgotamento sanitário separador absoluto, com condutos exclusivos para águas pluviais.

\section{Metodologia}

De acordo com Pereira et al. (2018), foi realizada um estudo de caso sobre a geração do escoamento superficial na área de estudo, por meio do uso do modelo matemático SWMM. 


\section{1 Área de estudo}

A Região Administrativa - RA do Riacho Fundo - RF I abrange uma população de 41.410 pessoas, onde 99,9\% dos domicílios tem acesso à rede geral da CAESB, 92,9\% possuem coleta e tratamento de esgoto e 99\% têm coleta de lixo (PDAD, 2019). Portanto, é uma RA com uma boa infraestrutura sanitária, onde a poluição difusa seria exclusivamente associada às águas do escoamento superficial, sem a presença de lançamento de esgoto clandestino, fato comprovado devido à falta de lâmina d'água nas redes de águas pluviais durante o período de estiagem.

Esta RA foi implementada em 1990 após o Governo do Distrito Federal instituir o programa habitacional para atender à demanda e organizar espacialmente o território, visando a erradicação de ocupações irregulares, loteando a granja Riacho Fundo (PDAD, 2019). A região tem predominância de casas unifamiliares, tendo a construção de prédio multifamiliares maiores que três andares a partir da década de 2010.

A região possui um área com 2,3 $\mathrm{km}^{2}$ e uma declividade razoável, variando de 0-12\%, com média de 3\% (Tsuji, 2018), no qual $46 \%$ é ocupada com áreas verdes e solo exposto, e 54\% por áreas construídas, dentre elas pavimentos, o que gera um CN médio de 70. O clima da região é o Tropical Savana, no qual a estação chuvosa se concentra de outubro a abril, com média de 1.540mm (Nunes, 2016). O tipo de solo predominante é o latossolo, mas já está bastante modificado devido a urbanização.

A estação hidrológica RF I está localizada no exutório das galerias de águas pluviais da RA Riacho Fundo I, as quais são lançadas, sem tratamento, no Riacho Fundo (Figura 1) que por sua vez é o tributário do Lago Paranoá considerado como o maior possuidor de cargas de poluentes (Aquino et al., 2018; Menezes, 2010). A estação é composta por um linígrafo de boia, réguas linimétricas e um pluviógrafos (Figura 2 a 5), outros dois estão alocados ao longo da área de estudo. Com esses dados, foi possível a realização da modelagem chuva-vazão. 
Figura 1 - Localização da RA RFI em relação a bacia do Riacho Fundo e ao Lago Paranoá com detalhe da rede de drenagem e do exutório com a localização da estação de monitoramento.

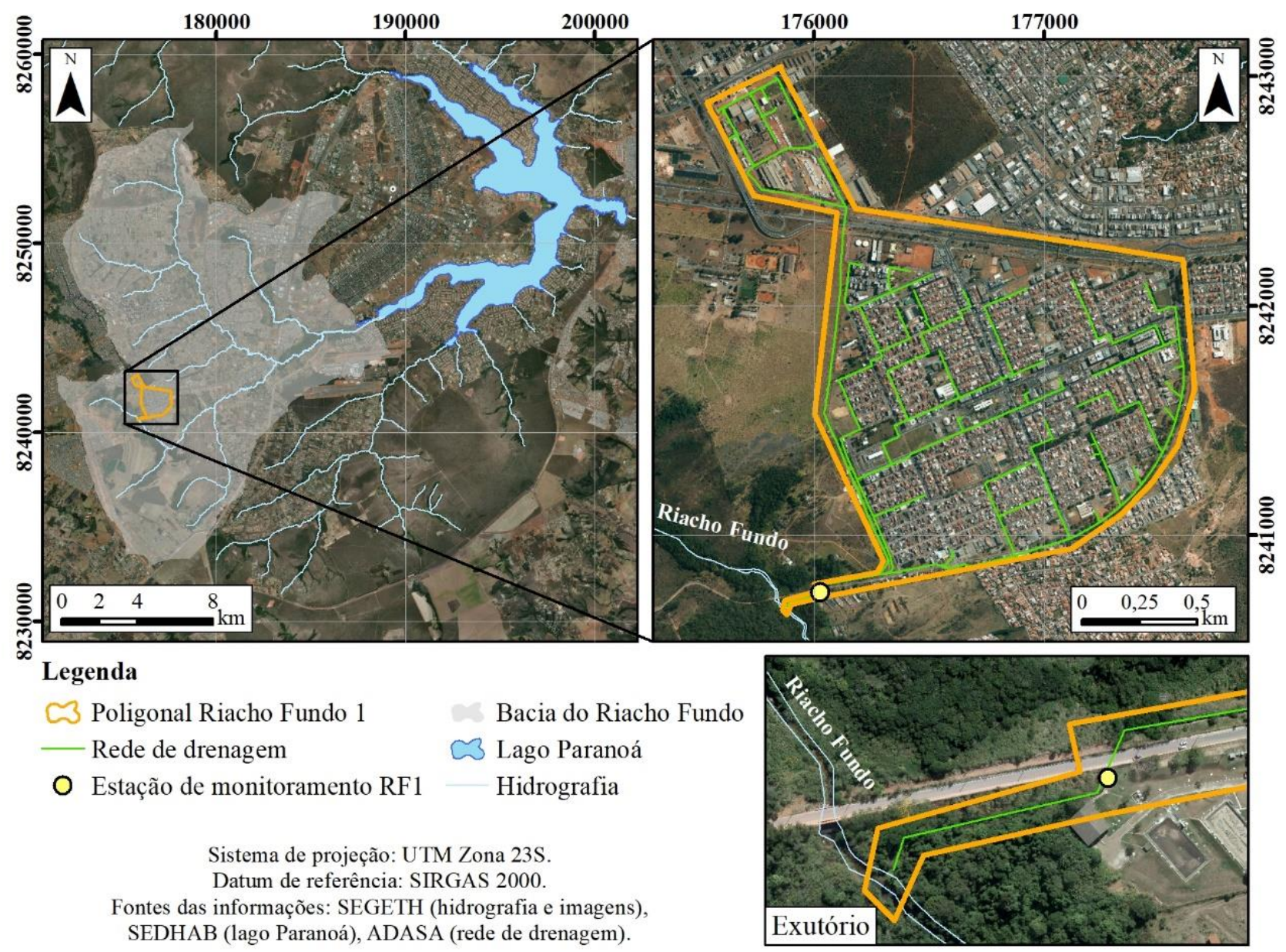

Fonte: Autores.

\begin{tabular}{|c|c|c|}
\hline $\begin{array}{c}\text { Figura 2 - Detalhe do Linígrafo } \\
\text { de boia instalado. }\end{array}$ & $\begin{array}{c}\text { Figura 3 - Réguas } \\
\text { Linimétricas. }\end{array}$ & $\begin{array}{c}\text { Figura 4 - Pluviógrafo da } \\
\text { estação RF I. }\end{array}$ \\
\hline & & \\
\hline
\end{tabular}


Figura 5 - Estação de monitoramento no exutório da Galeria de drenagem da bacia do RF I.

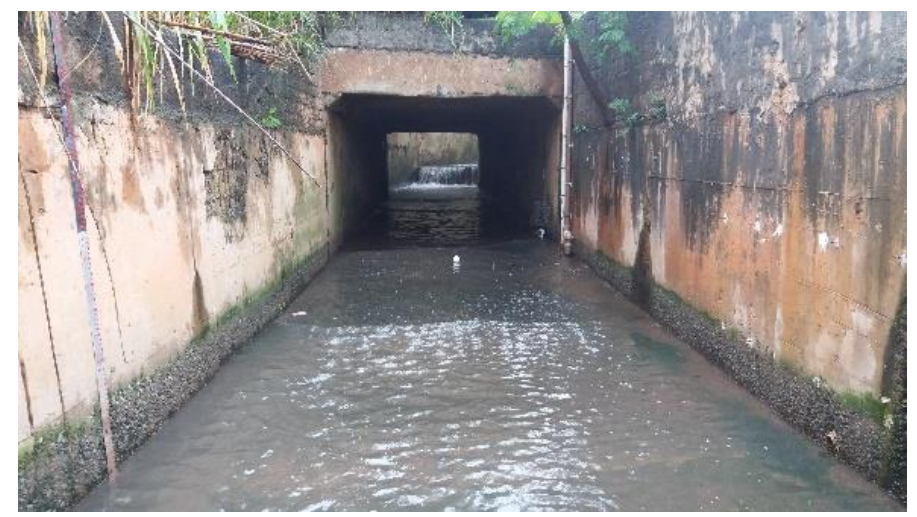

Fonte: Autores

\subsection{Modelagem Hidrodinâmica}

Foi utilizado o programa PCSWMM da CHIWater na versão 7.2 que corresponde ao modelo SWMM acoplado a um Sistema de Informação Geográfica - SIG, disponibilizado gratuitamente a instituições acadêmicas, bastante utilizado no DF para estudos de drenagem urbana (Costa, 2013; Souza, 2014; Tsuji et al., 2019; De Paula, 2019; Fileni et al., 2019; Brito et al., 2020).

A área de estudo foi dividida em sub-bacias de contribuição em que para cada uma delas o CN foi calculado por média ponderada, sendo utilizado o método SCS para geração do escoamento. Cada sub-bacia é considerado um reservatório não-linear com o escoamento superficial representado pela combinação das equações de Manning e da continuidade, gerado a partir da precipitação definida pelos 3 pluviógrafos pelo método do Polígono de Thiessen.

A rede de drenagem foi disponibilizada já georreferenciada pela ADASA baseada no Cadastro da NOVACAP (Figura 6). A rede possui informações quanto à localização dos poços de visita (PV), porém há ausência de dados como cota de entrada e cota de saída. Há também dados sobre dimensões e geometria de ramais, condutos e galerias, contudo há divergências quanto a outros dados, como comprimentos e declividade, que foram calculados ao longo do estudo.

Figura 6 - Rede de drenagem da RA RF I.

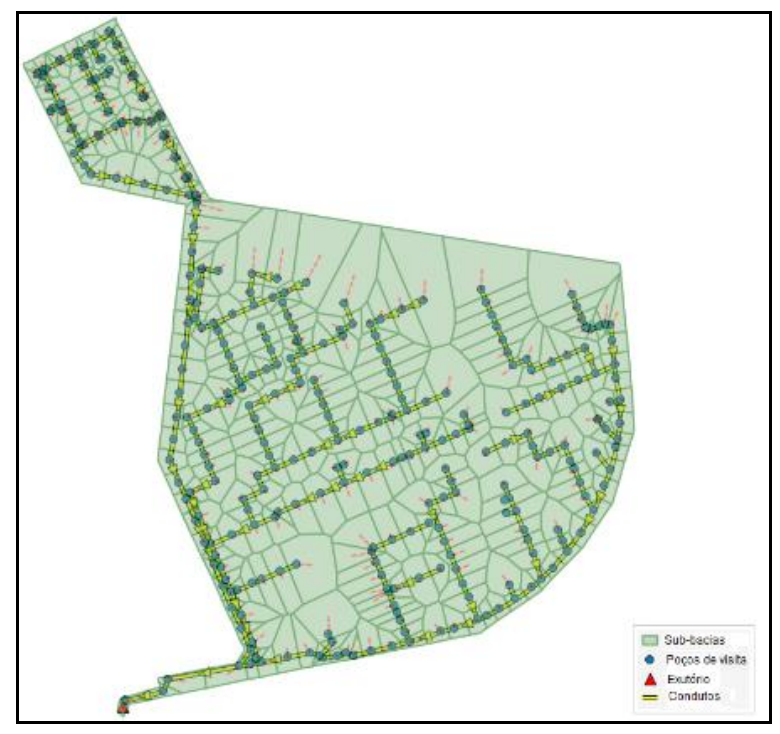

Fonte: Autores.

A bacia do RF I já havia sido área de estudo de Tsuji (2018), porém o mesmo não apresentou resultados satisfatórios, 
atribuído a problemas com os dados de precipitação e na curva-chave, que foram aperfeiçoados e, portanto, refeita a modelagem. Foram empregadas melhorias nos dados de entrada, como a nova série de precipitação, 2018-2019, composta por três pluviógrafos, bem como a vazão gerada na nova curva-chave do período, por meio da inclusão de mais pontos de medição de nível e vazão.

Para a simulação contínua foi necessário considerar a parcela de perda por evaporação, computada pelo método de Hargreaves. Esse método requer os dados diários de temperaturas mínima e máxima, obtidos pela estação climática do Inmet, além da irradiância solar extraterrestre e calor latente de vaporização, sendo esses dois últimos automaticamente computados pelo modelo (Rossman e Huber, 2016).

Para calibração e validação do modelo SWMM foi utilizado o método automático disponibilizado na versão do PCSWMM por meio da ferramenta SRTC (Sensitivity-based Radio Tuning Calibration), no qual a calibração é baseada na análise de incertezas dos parâmetros fornecido pelo usuário. A resposta calibrada é gerada pela interpolação linear dos valores do parâmetro, com base em 8 pontos de sensibilidade e o intervalo da incerteza. O resultado calibrado precisa ser avaliado, sendo necessário ainda a validação dos resultados para determinar os valores ótimos dos parâmetros.

Os parâmetros calibráveis são Área de contribuição das sub-bacias, Dstore perv - armazenamento em depressões permeáveis, $N$ Imperv - coeficiente de Manning para área impermeável, Width - largura de escoamento nas sub-bacias, Declividade das sub-bacias, $\mathrm{CN}$ - Curva número e $n$ - rugosidade dos canais da rede de drenagem e condutos, Cota de fundo e profundidade dos PVs. As porcentagens associadas à cada incerteza foram baseadas na recomendação de James (2005), conforme pode ser observado na Tabela 1 .

Tabela 1 - Porcentagem de alteração para o modelo.

\begin{tabular}{|l|l|l|}
\hline \multirow{5}{*}{ Sub-bacias } & Parâmetros & \% de Incerteza \\
\cline { 2 - 3 } & Área & $5-10 \%$ \\
\cline { 2 - 3 } & Width - Largura (m) & $50-100 \%$ \\
\cline { 2 - 3 } & Declividade (\%) & $5-10 \%$ \\
\cline { 2 - 3 } & N Imperv & $25-50 \%$ \\
\cline { 2 - 3 } & Dstore Perv & $25-50 \%$ \\
\cline { 2 - 3 } & CN & $50-100 \%$ \\
\hline PV & Cota de fundo & $5-10 \%$ \\
\cline { 2 - 3 } & Profundidade & $5-10 \%$ \\
\hline Condutos & Coef. Manning & $50-100 \%$ \\
\hline
\end{tabular}

Fonte: Autores.

A partir dos dados de chuva e vazão, foi possível analisar os eventos ocorridos na bacia no período estudado e descartado os períodos com falhas. Identificados os eventos, optou-se por calibrar cada um deles, foram selecionados os 3 que obtiveram melhores valores para as funções objetivos, e em seguida realizou-se a etapa de validação. Ao final, foi selecionado o evento dentre os três que apresentou os melhores valores para as funções objetivos na validação.

As funções objetivos selecionadas foram: ISE rating e ISE (Integral Square Error - integral do erro quadrático), NSE(coeficiente de Nash-Sutcliffe), R² (coeficiente de determinação), SEE (Standard Error of Estimate - erro padrão da estimativa), LSE (Simple Least Square Regression) e RMSE (Root Mean Square Error - raiz quadrada do erro médio).

\section{Resultados e Discussão}

Devido a limitação da extrapolação da curva-chave, o que gera uma grande incerteza dos valores de vazões quando altas, resolveu-se simular o modelo chuva-vazão somente nos eventos cuja vazão calculadas pela curva-chave não ultrapasse 1,5 vezes as vazões medidas, o que indica um limite de vazões de $5,25 \mathrm{~m}^{3} / \mathrm{s}$, totalizando 21 eventos de estudo. 


\subsection{Calibração do Modelo Chuva-Vazão SWMM da bacia RF I}

O modelo da bacia do RF 1 já havia sido estudado por Tsuji (2018) com eventos ocorridos entre 2017 e 2018. Nesta pesquisa foram utilizados os eventos ocorridos no ano hidrológico 2018-2019 com a incorporação dos dados de um novo pluviográfo na bacia de estudo.

Além disso, foi realizado a modelagem contínua entre outubro/2018 até maio/2019, ou seja, houve a inclusão de perda por evapotranspiração por meio do método de Hargreaves, que permite um melhor detalhamento do ciclo hidrológico na bacia, porém a análise do modelo foi realizada para cada evento.

Na Figura 7 é possível visualizar o resultado do escoamento superficial gerado pelo modelo SWMM sem calibração, somente com a inserção dos dados de entrada, para cada evento monitorado. Percebe-se que o modelo tem uma razoável resposta aos eventos de chuva simulado, porém ainda deficiente, que foi aprimorada com a calibração do modelo.

Figura 7 - Representação dos 21 Hidrogramas gerado pelo modelo SWMM para a bacia do RF1 sem calibração.
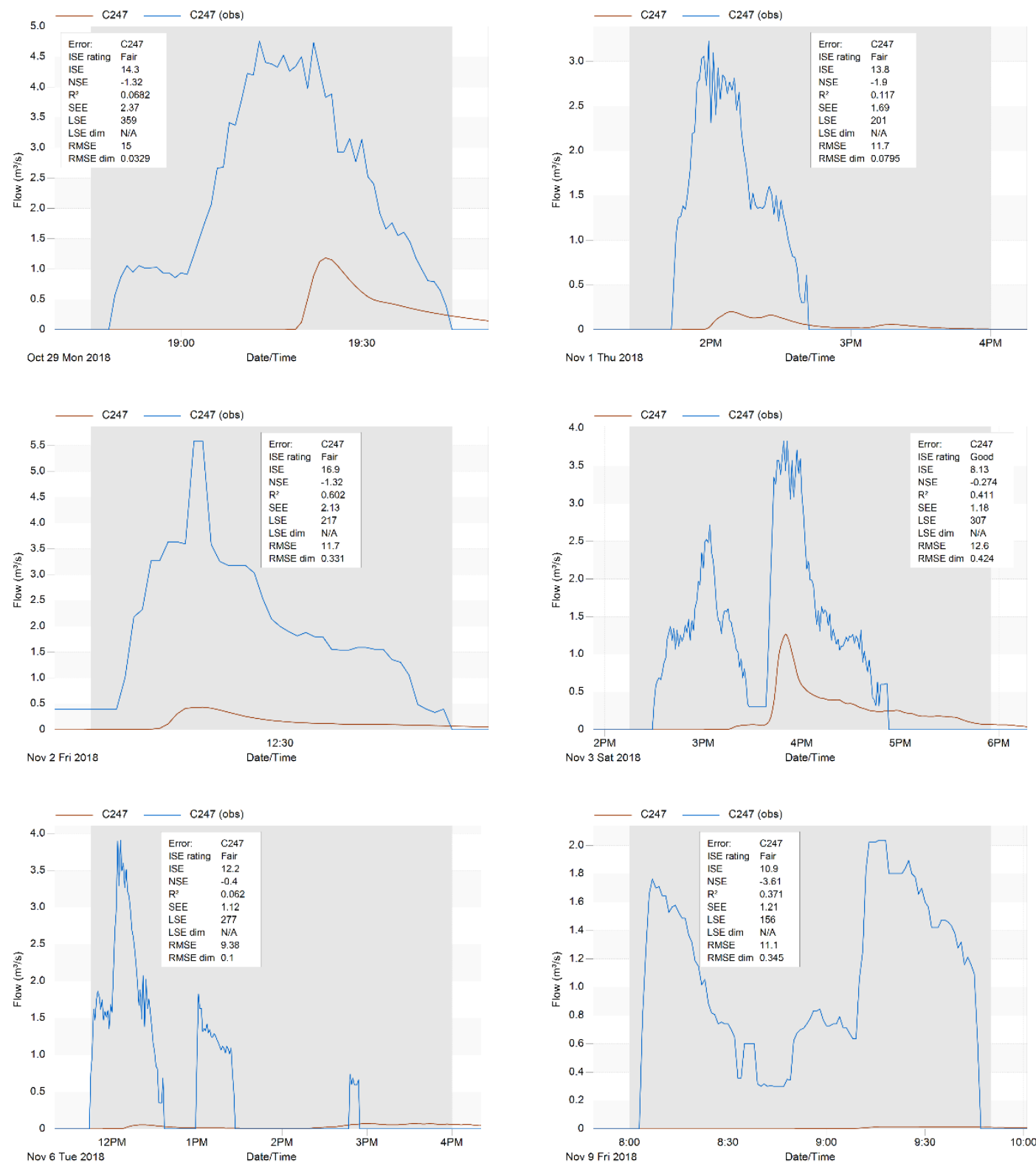
Research, Society and Development, v. 10, n. 1, e6010111458, 2021

(CC BY 4.0) | ISSN 2525-3409 | DOI: http://dx.doi.org/10.33448/rsd-v10i1.11458
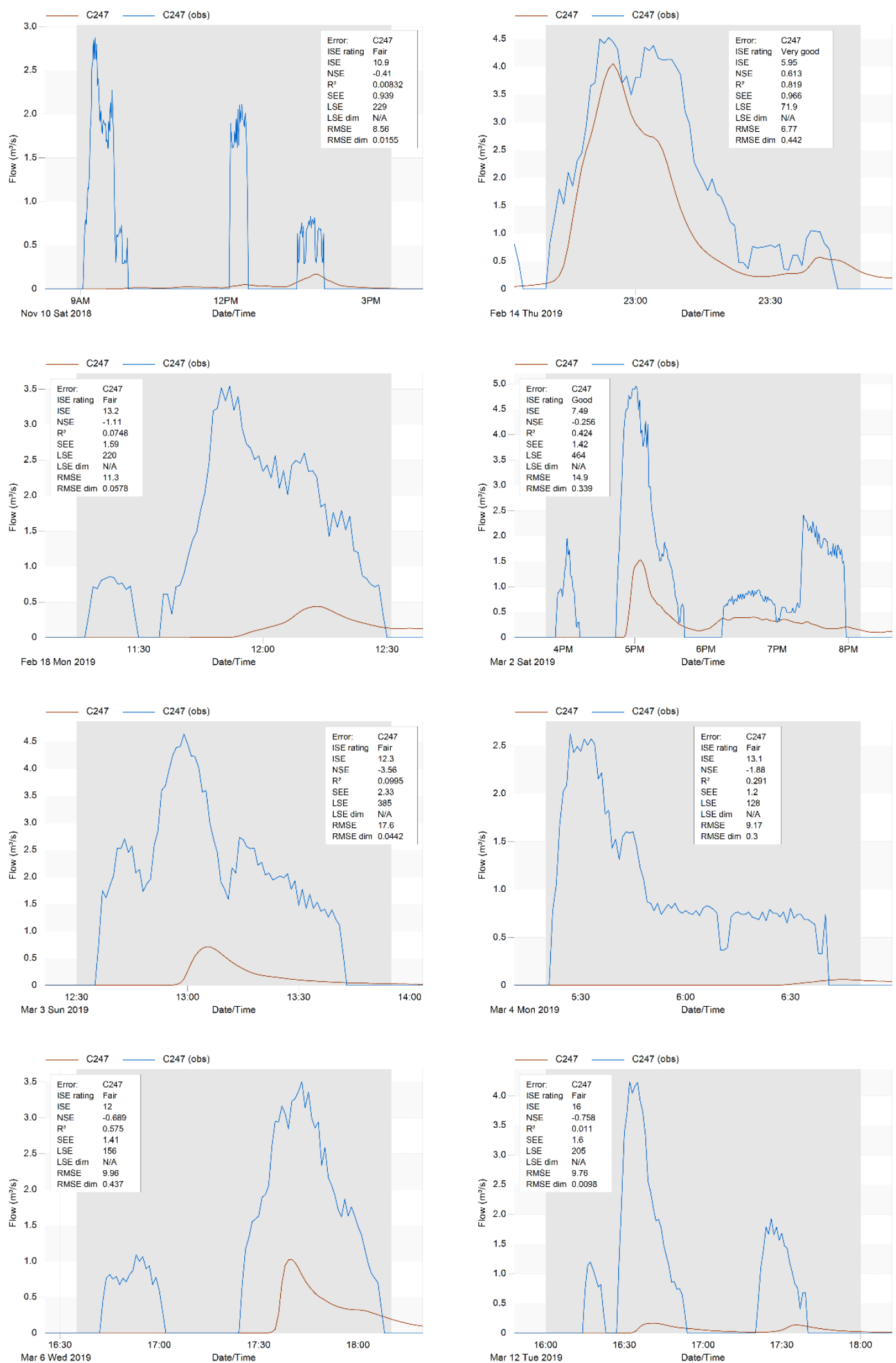

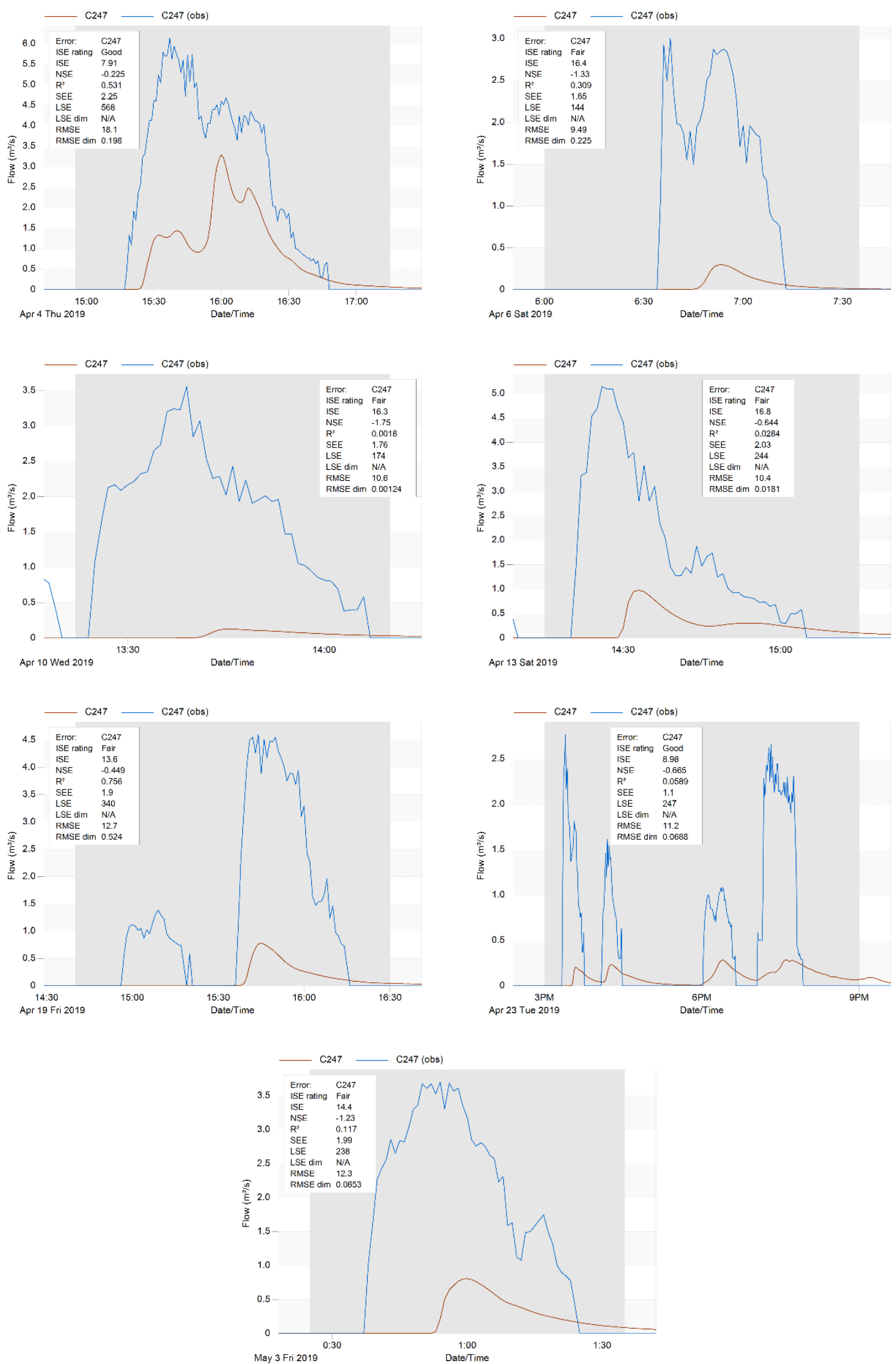

Fonte: Autores

Compreendendo a necessidade de calibração do modelo, foi utilizado a ferramenta SRTC que seleciona a alternativa ótima para cada evento. O evento que obteve os melhores resultados para as funções objetivos correspondeu ao do dia 12 de março de 2019 (Tabela 2 e Fonte: Autores. 
Figura 8). O evento calibrado apresentou o comportamento de geração do escoamento mais próximo do real, sendo também o que apresentou os melhores resultados para validação do modelo.

Tabela 2 - Comparação da calibração do evento 12/03/19 no modelo SWMM.

\begin{tabular}{|l|l|l|}
\hline Parâmetros & Sem calibração & Com Calibração \\
\hline ISE rating & Fair (Razoável) & Muito bom \\
\hline ISE & 16.1 & 4.64 \\
\hline NSE & -0.747 & 0.851 \\
\hline R $^{2}$ & 0.0005 & 0.865 \\
\hline SEE & 1.6 & 0.466 \\
\hline LSE & 206 & 17.4 \\
\hline RMSE & 9.74 & 3.28 \\
\hline RMSE dim & 0.000418 & 0.77 \\
\hline
\end{tabular}

Fonte: Autores.

Figura 8 - Hidrograma do evento do dia 12/03/19 calibrado.

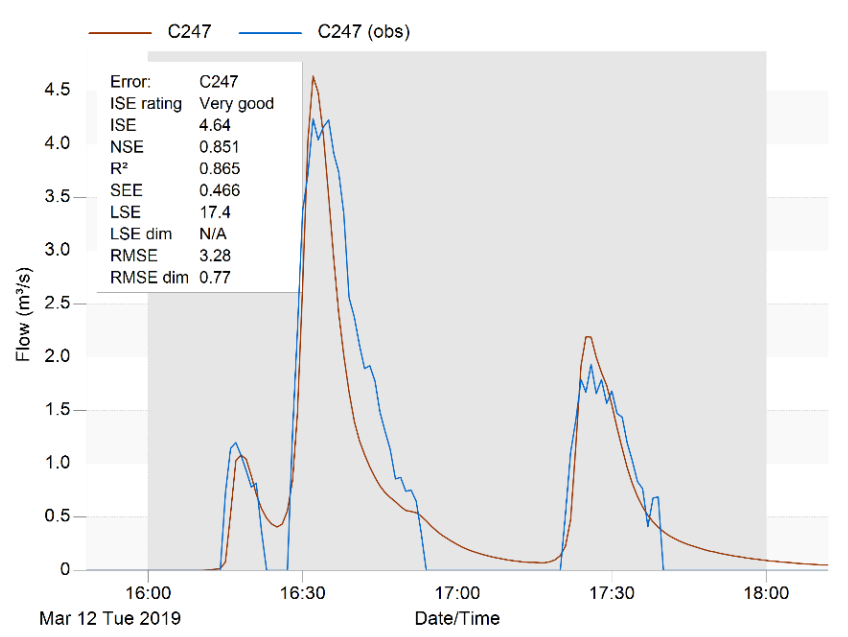

Fonte: Autores.

O modelo calibrado apresentando na Fonte: Autores.

Figura 8 foi considerado satisfatório, tendo em vista que obteve NSE $=0,851, \mathrm{R}^{2}=0,865$, ISE $=4,64$, dentre as outras funções objetivos com resultados bons, representando pequenas divergências entre os valores observados e os simulados. Para essa solução, foi necessário que o CN fosse acrescido em 62\%, e que a área se ajustassem em 8,5\%, e as cotas de fundo dos PVs em 8,3\%, enquanto houve uma redução de $100 \%$ do n, sendo o calibrado de 0,010 , e também $100 \%$ no Dstore Perv, sendo o valor final entre 0,099 - 1,35.

\subsection{Validação do Modelo Chuva-Vazão SWMM da bacia RF I}

Com o modelo calibrado para o evento do dia 12/03/19, esse modelo foi usado para avaliar os demais eventos monitorados (

Figura 9). Em todos os eventos verificados houve uma melhora na aproximação do hidrograma aos dados observados, confirmados pelos resultados das funções objetivos. 
Figura 9 - Validação do modelo calibrado para o evento do dia 12/03/19 para os demais 20 eventos monitorados.
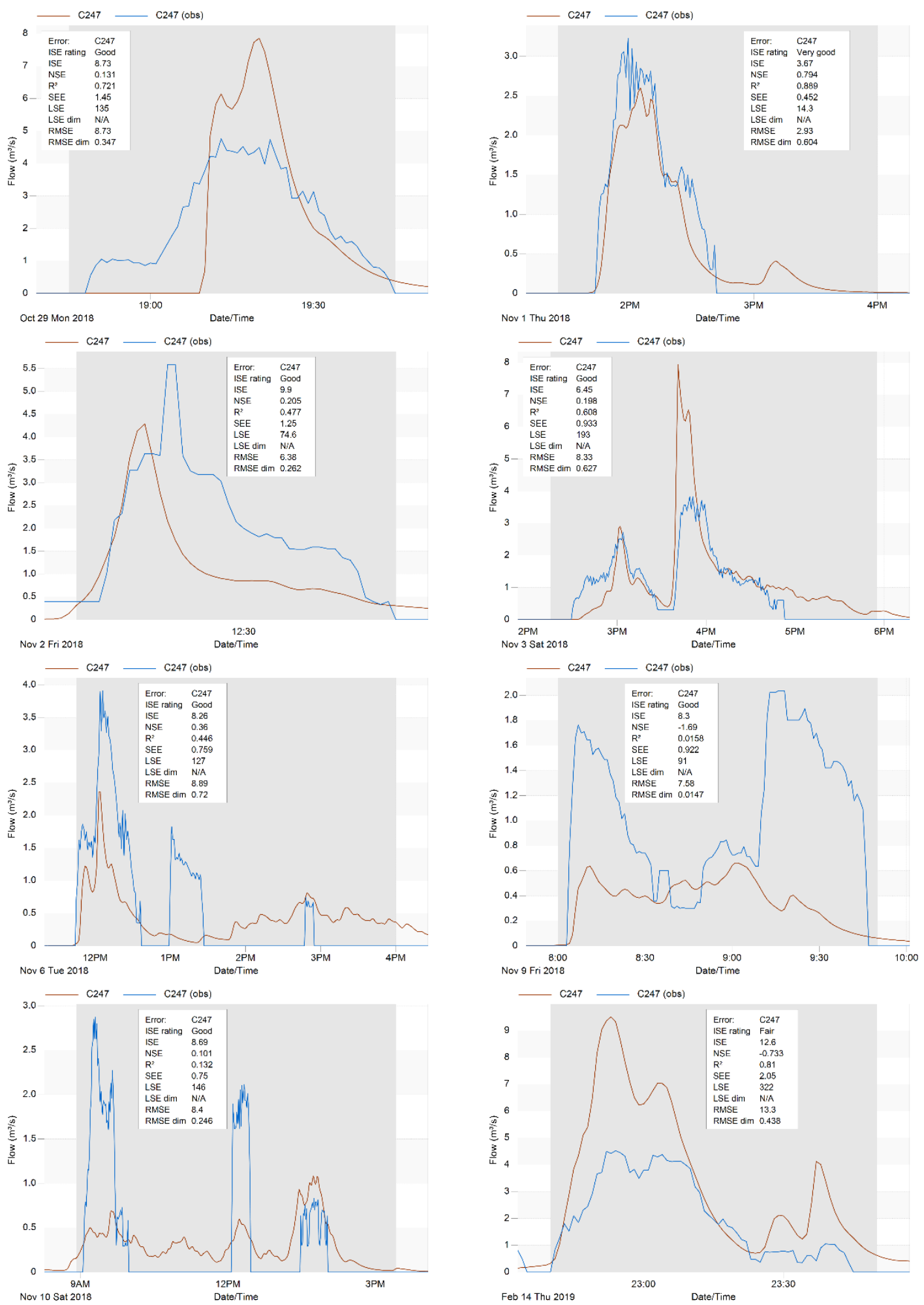
Research, Society and Development, v. 10, n. 1, e6010111458, 2021

(CC BY 4.0) | ISSN 2525-3409 | DOI: http://dx.doi.org/10.33448/rsd-v10i1.11458
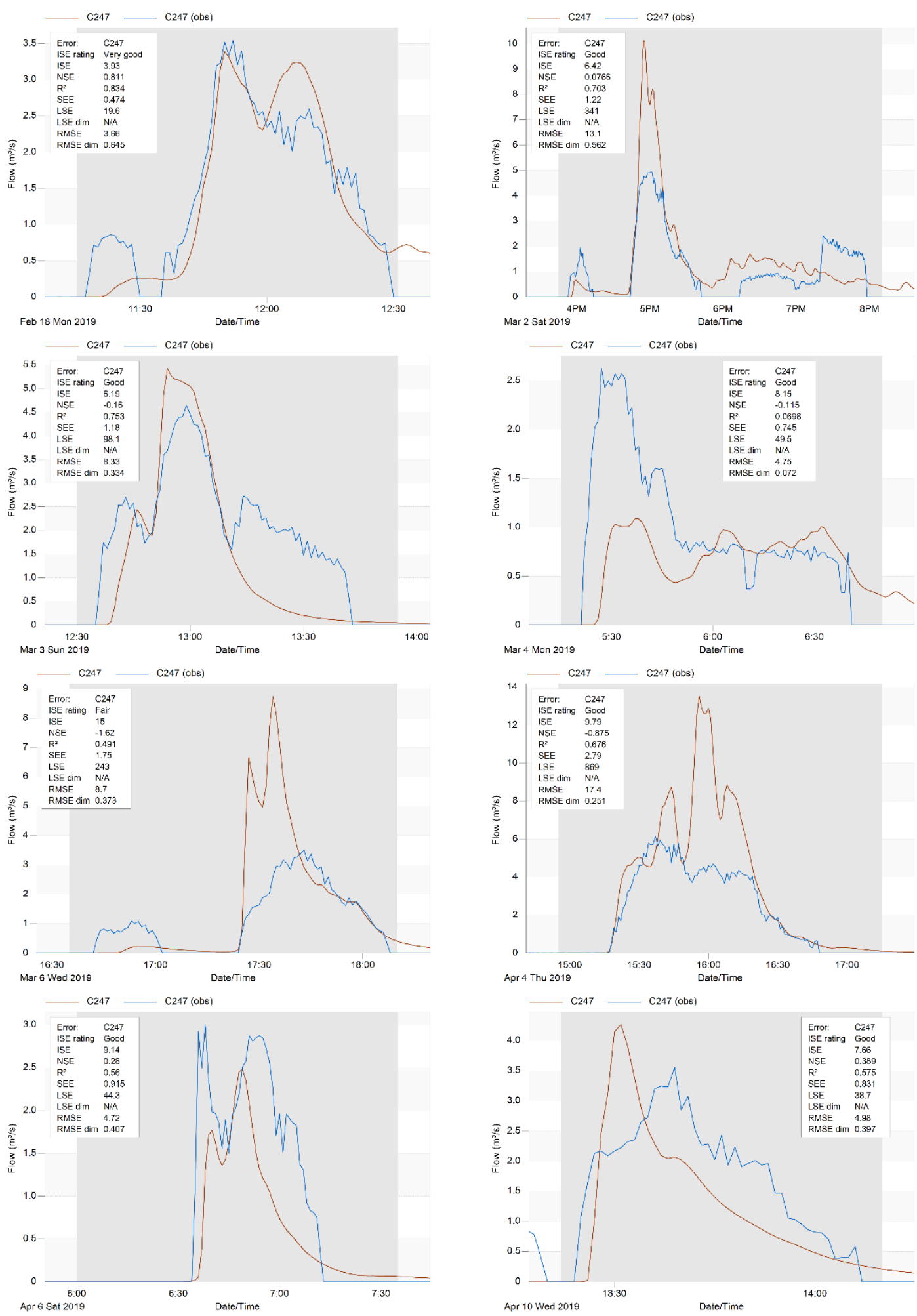

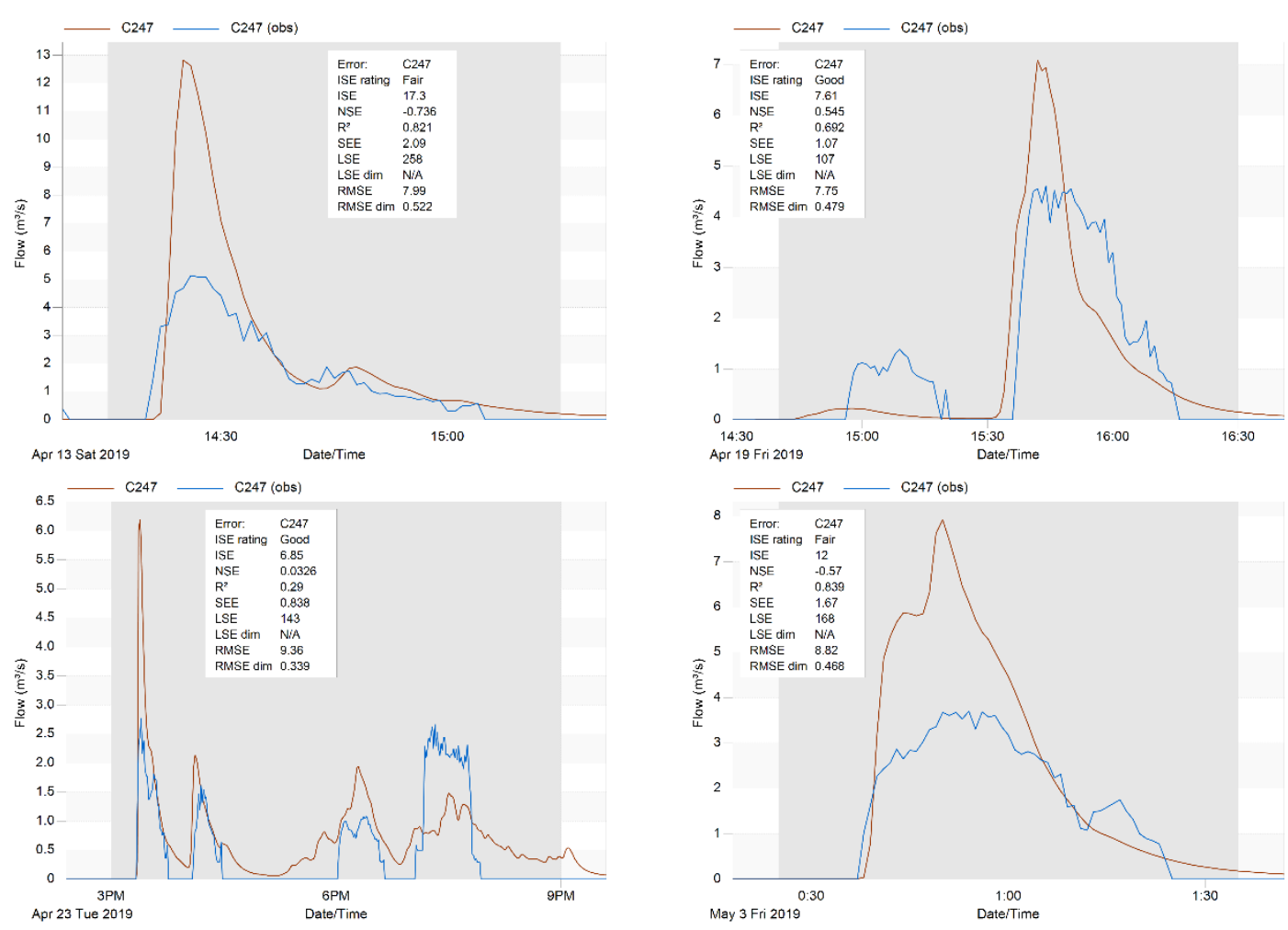

Fonte: Autores.

Em apenas três eventos, não foi possível resultados considerados "BOM" (Good) e com valores de NSE positivos, são os eventos cujo modelo gerou vazões muito altas, com $Q_{\text {pico }}$ maiores que $8 \mathrm{~m}^{3} / \mathrm{s}$, o que pode ser associado a problemas nos dados monitorados, devido à dificuldade de medição de grandes vazões, refletidos na curva-chave. Nos outros eventos, os valores do ISE são "BOM” (Good), porém ainda se observa NSE $<0$ em 40\% dos eventos, onde a Qpico é muito superestimada, no qual o crescimento da vazão no modelo é muito exagerado, fazendo com que as funções objetivos ficassem com valores fora do intervalo considerado adequado.

Tsuji (2018) fez a modelagem chuva-vazão para mesma bacia em 8 eventos, e somente em 2 deles o NSE foi positivo, que foi justamente nos eventos onde os volumes precipitados foram semelhantes, enquanto que em 7 eventos o ISE foi considerado razoável ou ruim.

Yazdi et al. (2019) ratifica que o modelo SWMM é um dos mais utilizado no mundo para avaliação dos impactos causados em águas receptoras devido a urbanização, justamente porque tem como mais sensíveis os parâmetros relacionados com a impermeabilização, ou seja, se adequam bem a bacias urbanas. No geral, os modelos no SWMM conseguem reproduzir bem a vazão de pico e o volume do escoamento gerado, como confirmado por Kourtis et al. (2017), Shinma (2015), Costa (2013), Souza (2014), Garcia et al (2006).

\section{Considerações Finais}

A partir dos dados de monitoramento de precipitação e vazão na bacia do RF I, é possível concluir que o modelo SWMM do RF I obteve resultados mais semelhantes a realidade após a sua calibração, sendo esta etapa fundamental para utilização de modelos hidrológicos e hidráulicos no melhor gerenciamentos das águas pluviais.

O modelo SWMM necessitou de ajustes em parâmetros como área, cota de fundo dos PVs, e principalmente no coeficiente de Manning n, que refere-se sobre a rugosidade dos condutos, no Dstore Perv que se refere sobre o armazenamento em áreas permeáveis e o CN que é um indicador da impermeabilização nas sub-bacias. 
Deve-se utilizar mais de uma função objetivo, pois apesar de que em $40 \%$ dos eventos validados houve NSE negativos, o modelo ainda se mostrou satisfatório em decorrências de outras métricas estatísticas. O problema no NSE ocorre devido ao superdimensionamento do modelo em relação as Qpico, que pode ser atribuído a problemas na geração da curvachave, que precisa ser aperfeiçoada, evidenciando a relevância dos dados de monitoramento para a qualidade dos resultados de modelagem.

A partir da modelagem realizada para o desenvolvimento dessa pesquisa, também foi possível aprofundar os conhecimentos sobre hidrologia no sistema de drenagem urbana da RA Riacho Fundo I, com a inclusão da perda por evaporação na modelagem da bacia.

A calibração de modelos é um processo complexo que exige entendimento das premissas assumidas, sendo os dados de entradas essenciais para que o modelo possa reproduzir o mais próximo da realidade a transformação da chuva em vazão.

Recomenda-se ainda que sejam realizados estudos para aplicação do medidas compensatórias a fim de reduzir os impactos causados pelas grandes vazões e pela poluição difusa dessas águas pluviais.

\section{Agradecimentos}

Agradecemos a CAESB por permitir o acesso a ETE Riacho Fundo I. A ANA, CAPES, FAPDF, FUNAPE e FINATEC pelo apoio à pesquisa ao qual o trabalho está associado.

\section{Referências}

Aquino, I., Roig, H. L. Oliveira, E. Garnier, J., Guimarães, E., \& Koide, S. (2018). Variação temporal da descarga sólida em suspensão e identificação de minerais a partir de aperfeiçoamento de método de amostragem automática no Córrego Riacho Fundo, Brasília, Distrito Federal. Geologia USP. Série Científica, 18(2), 171-185.

Barros, A., Neves, M. G. F. P., \& Henrique, D.C. (2016). Variabilidade E Causas Na Geração De Vazão Em Bacias Urbanas: Estudo De Caso Do Riacho Do Sapo, Maceió-AL. Revista Eletrônica de Gestão e Tecnologias Ambientais. 4, 220.

Blumensaat, F. \& Wolfram, M. \& Krebs, P. (2012). Sewer model development under minimum data requirements. Environ. Earth Sci., 65(5), $1427-1437$.

Brito, L. K. S., Costa, M. E. L. \& Koide, S. (2020). Assessment of the Impact of Residential Urban Patterns of Different Hillslopes on Urban Drainage Systems and Ecosystem Services in the Federal District, Brazil. Sustainability, 12(14), 5859.

Broekhuizen, I., Leonhardt, G., Marsalek, J. \& Viklander, M. (2019). Calibration event selection for green urban drainage modelling. Hydrology and Earth System Sciences Discussions.

Chung, E.-S. \& Hong, W.-P. \& Lee, K. S. \& Burian, S. J. (2011). Integrated use of a continuous simulation model and multi-attribute decision making for ranking urban watershed management alternatives." Water Resour. Manage, 25(2), 641-659.

Costa, M. E. L (2013). Monitoramento e modelagem das águas da drenagem urbana na bacia do lago Paranoá. Dissertação de Mestrado em Tecnologia Ambiental e Recursos Hídricos, Publicação PTARH.DM-148/2013, Departamento de Engenharia Civil e Ambiental, Universidade de Brasília, Brasília, DF, 179 p.

De Paula, A. C. V. (2019). Estudo experimental e modelagem da lagoa de detenção do guará - DF: comportamento no amortecimento de cheias e na alteração da qualidade da água. Dissertação De Mestrado Em Tecnologia Ambiental E Recursos Hídricos da Universidade de Brasília.

Fileni, F. Costa, M. E. L. \& Alves, C. M. A. (2019). The application of LIDs in Savanna region for mitigation of flooded areas. RBRH. 24.

Garcia, J. I. B. \& Paiva, E. M. C. D. (2006). Monitoramento Hidrológico E Modelagem da Drenagem Urbana da Bacia do Arroio Cancela - RS. Revista Brasileira de Recursos Hídricos, 11(4), 99-108.

James, W. (2005). Rules for responsible modeling (4a ed.). Computational Hydaulics International.

Kourtis, I. M. Kopsiaftis, G., Bellos, V., \& Tsihrintzis, V. A. (2017). Calibration and validation of SWMM model in two urban catchments in Athens, Greec. Proceedings 15th International Conference on Environmental Science and Technology Rhodes, Greece.

Lian, Q., Yao, L., Uddin Ahmad, Z., Lei, X., Islam, F., Zappi, M. E., \& Gang, D. D. (2019), Nonpoint source pollution. Water Environ Res, 91, $1114-1128$.

Menezes, P. H. J. (2010). Avaliação do efeito das ações antrópicas no Processo de escoamento superficial e Assoreamento na bacia do Lago Paranoá. Dissertação De Mestrado. Universidade de Brasília - UnB do Instituto de Geociências - IG.

Müller, A., Österlund, H., \&Marsalek, J. (2019). The pollution conveyed by urban runoff: A review of sources, Science of the Total Environment. 
Research, Society and Development, v. 10, n. 1, e6010111458, 2021

(CC BY 4.0) | ISSN 2525-3409 | DOI: http://dx.doi.org/10.33448/rsd-v10i1.11458

Niazi, M., Nietch, C., Maghrebi, M., Jackson, N., Bennett, B., Tryby, M. \& Massoudieh, A. (2017). Storm Water Management Model: Performance Review and Gap Analysis. J. Sustainable Water Built Environ. 3.

Nunes, G. (2016). Aplicação do Modelo Swat no Estudo Hidrológico e de Qualidade da Água da Bacia do Lago Paranoá - DF. Dissertação de Mestrado em Tecnologia Ambiental e Recursos Hídricos Departamento de Engenharia Civil e Ambiental. 148pg.

Paule-Mercado C., \& Lee, C. (2017). Calibration of the SWMM for a mixed land use catchment in Yongin, South Korea. Desalination And Water Treatment. 63. 381-388.

PDAD - Pesquisa Distrital por Amostra de Domicílios 2018. (2019). Governo Do Distrito Federal. Companhia De Planejamento Do Distrito Federal CODEPLAN.

Pereira, A. S., Shitsuka, D. M., Parreira, F. J., \& Shitsuka, R. (2018). Metodologia da pesquisa científica. [e-book]. Santa Maria. Ed. UAB/NTE/UFSM.

Pompêo, C. A (1999). Development of a state policy for sustainable urban drainage. Urban Water,1, 155-160.

Porto, M. F. (1995). Aspectos qualitativos do escoamento superficial em áreas urbanas. In: Drenagem Urbana. Porto Alegre: ABRH e UFRGS, $387-414$.

Rossman, L. A. (2009). Storm Water Management Model User's Manual Version 5.0. Cincinnati: U. S. Environmental Protection Agency. USA.

Rossman, L. A., \& Huber, W. C. (2016). Storm Water Management Model Reference Manual Volume I - Hydrology. Cincinnati (EUA): USEPA (United States Environment Protection Agency), 2016.

Shinma, T. A. (2015). Avaliação de incertezas na calibração automática do modelo SWMM. Tese de Doutorado em Hidráulica e Saneamento, Escola de Engenharia de São Carlos, Universidade de São Paulo, São Carlos, SP, 212.

Silveira, A. L. L. (1998). Drenagem urbana: gerenciamento, simulação, controle. In: Hidrologia urbana no Brasil. Porto Alegre: Editora da UFRGS/ABRH, $8-25$.

Souza, F. P. (2014). Monitoramento e Modelagem Hidrológica da Sub-bacia do Lago Paranoá - Brasília/DF - e Avaliação de Bacias de Detenção. Dissertação de Mestrado em Tecnologia Ambiental e Recursos Hídricos. Faculdade de Tecnologia. Departamento de Engenharia Civil e Ambiental. Universidade de Brasília, Brasília, DF, 139p.

Tsuji, T. M. (2018). Monitoramento e Modelagem das Águas de Drenagem Pluvial no Núcleo Urbano da Bacia do Ribeirão Riacho Fundo, Brasília - DF. Dissertação De Mestrado Em Tecnologia Ambiental E Recursos Hídricos da Universidade de Brasília.

Tsuji, T. M., Costa, M. E. L., \& Koide, S. (2019). Diffuse pollution monitoring and modelling of small urban watershed in Brazil Cerrado. Water Science and Technology, 79, 1912-1921.

Walsh, T. C., \& Pomeroy, C. A., \&Burian, S. J. (2014). Hydrologic modeling analysis of a passive, residential rainwater harvesting program in an urbanized, semi-arid watershed. J. Hydrol., 508, 240-253

Wu, J., Thompson, J., Kolka, R., Franz, K., Stewart, T. (2013). Using the Storm Water Management Model to predict urban headwater stream hydrological response to climate and land cover change. Hydrol. Earth Syst. Sci. 2013, 17, 4743-4758.

Xu, Z., Xiong, L., \& Li, H. (2019). Runoff simulation of two typical urban green land types with the Stormwater Management Model (SWMM): sensitivity analysis and calibration of runoff parameters. Environ Monit Assess 191, 343.

Yazdi, M. N., Ketabchy, M., Sample, D. J., Scott, D., \& Liao, H. (2019). An evaluation of HSPF and SWMM for simulating streamflow regimes in an urban watershed. Environmental Modelling \& Software, 118, 211-225.

Zhang, Y., Zhao, W. Chen, X., Jun, C., Hao, J. Tang, X., \& Zhai, J. (2020). Assessment on the Effectiveness of Urban Stormwater Management. Water 2020, 13,4 . 